\title{
Tubular structures of GaS
}

\author{
Th. Köhler, ${ }^{1}$ Th. Frauenheim, ${ }^{1}$ Z. Hajnal, ${ }^{1}$ and G. Seifert ${ }^{2}$ \\ ${ }^{1}$ Theoretische Physik, Universität Paderborn, 33098 Paderborn, Germany \\ ${ }^{2}$ Institut für Physikalische Chemie, Technische Universität Dresden, 01062 Dresden, Germany
}

(Received 11 December 2003; published 13 May 2004)

\begin{abstract}
In this Brief Report we demonstrate, using density-functional tight-binding theory, that gallium sulfide (GaS) tubular nanostructures are stable and energetically viable. The GaS-based nanotubes have a semiconducting direct gap which grows towards the value of two-dimensional hexagonal GaS sheet and is in contrast to carbon nanotubes largely independent of chirality. We further report on the mechanical properties of the GaS-based nanotubes.
\end{abstract}

DOI: 10.1103/PhysRevB.69.193403

PACS number(s): 73.22.-f, 71.15.Nc, 61.46.+w, 62.25.+g

Since the first successful synthesis of carbon nanotubes by Iijima $^{1}$ in 1991, the worldwide interest in those lowdimensional nanosized materials is unbroken until now. Due to their extraordinary physical properties promising technical applications become possible. During the last decade considerable effort has been invested into controlling properties of nanotubes, for example, diameter, chirality, and structural defects during the synthesis; we refer the reader to a paper of Dai in Ref. 2, and references within. Together with the rapid development of microscopy techniques such as transmission electron microscopy, scanning tunneling microscopy, or atomic force microscopy powerful tools for manipulating the world of nanometers are available now. This new techniques allow experimental determination and measurements of properties and their goal-directed change itself. So one major field of current nanotube research is the functionalization of nanotubes to prepare for advanced technological solutions as nanoscale devices. ${ }^{3-5}$

Simultaneously with pure carbon nanotubes there is a growing interest in studying possible tubular structures of inorganic materials. Using chemical analogy between graphite and two-dimensional (2D) layered inorganic compounds Tenne and co-workers ${ }^{6}$ successfully produce metalchalcogenide nanotubes $X-\mathrm{S}_{2}(X=\mathrm{Mo}, \mathrm{W})$ and their related selenides. Mostly among the layered inorganic compounds are those which can form fullerenelike structures, too, see, for example, $\mathrm{MoS}_{2}$ as an inorganic candidate. ${ }^{6,7}$ So up to now tubular structures of bismuth $(\mathrm{Bi}),{ }^{8}$ gallium nitride $(\mathrm{GaN}),{ }^{9}$ boron nitride $(\mathrm{BN}),{ }^{10}$ niobium diselenide $\mathrm{NbS}_{2},{ }^{11}$ and as well oxidic tubes of $\mathrm{V}_{2} \mathrm{O}_{5}$ (Ref. 12) have been experimentally prepared. For a recent review about nanotubes of inorganic materials see, e.g., Tenne and Zettl in Ref. 2.

In 1998 the possible formation of gallium selenide (GaSe) nanotubes have been studied by Cote et $a l .{ }^{13}$ using an $a b$ initio plane-wave pseudopotential method on the basis of local-density approximation. As one conclusion of their GaSe calculations they proposed also the (probable) formation of stable gallium sulfide $(\mathrm{GaS})$ nanotubes. Within this work we will bring into focus these suggestions. Nowadays a variety of chemical routes to crystalline and amorphous gallium sulfide modifications are known. One route to generate gallium sulfide thin films is a chemical vapor deposition process of molecular organic precursors containing bonding information about the phase later to grow. In Refs. 14 and 15 it has been demonstrated how to use the family of tert-butyl gallium sulfide compounds to produce crystalline hexagonal $\mathrm{GaS}$ from $\left[\left({ }^{\mathrm{t}} \mathrm{Bu}\right)_{2} \mathrm{Ga}\left(\mathrm{S}^{\mathrm{t}} \mathrm{Bu}\right)\right]_{2}$, amorphous $\mathrm{GaS}$ from $\left[\left({ }^{\mathrm{t}} \mathrm{Bu}\right) \mathrm{GaS}\right]_{7}$ and cubic $\mathrm{GaS}$ from $\left[\left({ }^{\mathrm{t}} \mathrm{Bu}\right) \mathrm{GaS}\right]_{4}$, respectively. Additionally the cubic phase of $\mathrm{GaS}$ is of interest for surface passivation applications in GaAs based electronic devices because of their isostructural good lattice matching to GaAs. ${ }^{16}$ Perhaps gallium sulfide tubular structures can be found and used for novel device solutions.

To obtain the structure, energetics, and electronical and mechanical properties for possibly existing GaS-based nanotubes we performed atomistic simulations within a selfconsistent charge-density-functional based nonorthogonal tight-binding scheme (SCC-DFTB). ${ }^{17,18}$ Up to now a wide range of possible questions could be answered by usage of this method. We refer the reader to recent studies on silicon based tubular structures, ${ }^{19}$ the discussion of properties of $\mathrm{MoS}_{2}$ (Ref. 7), and exofluorinated carbon nanotubes, ${ }^{20}$ as examples.

Experimentally it is known that hexagonal GaS has a layered $[\ldots \mathrm{S}-\mathrm{Ga}-\mathrm{Ga}-\mathrm{S} \ldots]$ repeating unit built by sixmembered $\mathrm{Ga}_{3} \mathrm{~S}_{3}$ rings. Our DFTB calculations predict a stable hexagonal double-layered structure, shown in Fig. 1, with bond distances of $2.48 \AA$ and $2.37 \AA$ for the Ga-Ga and $\mathrm{Ga}-\mathrm{S}$ bond length, respectively. The bond angle for Ga-Ga-S is $115.76^{\circ}$ and for the S-Ga-S angle we find $102.51^{\circ}$. In order to study the energetic viability of the corresponding $\mathrm{GaS}$ nanotubes, as well as to determine their possible structures and to characterize their electronic and mechanical properties, we have performed a series of calculations in
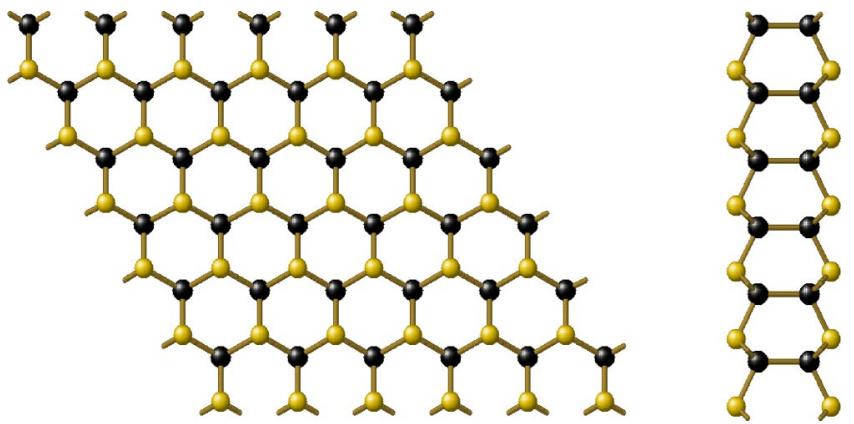

FIG. 1. The structure (top view-left figure, side view-right) of a hexagonal GaS layer as predicted by our DFTB calculations. 
$(30,0) \mathrm{GaS}$

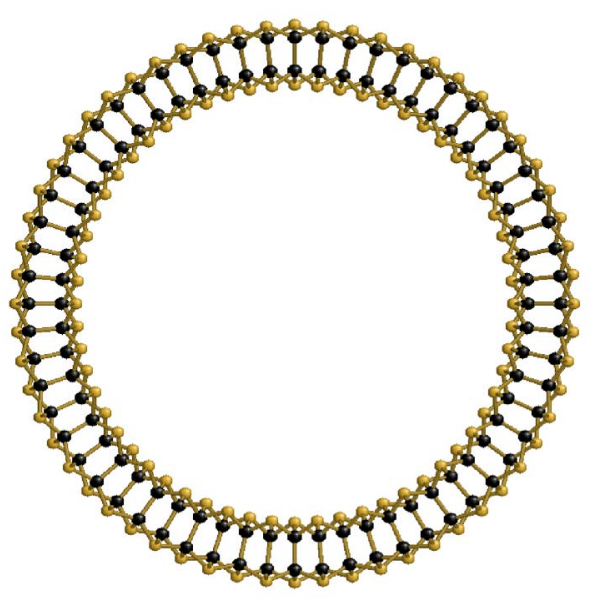

$(20,20) \mathrm{GaS}$

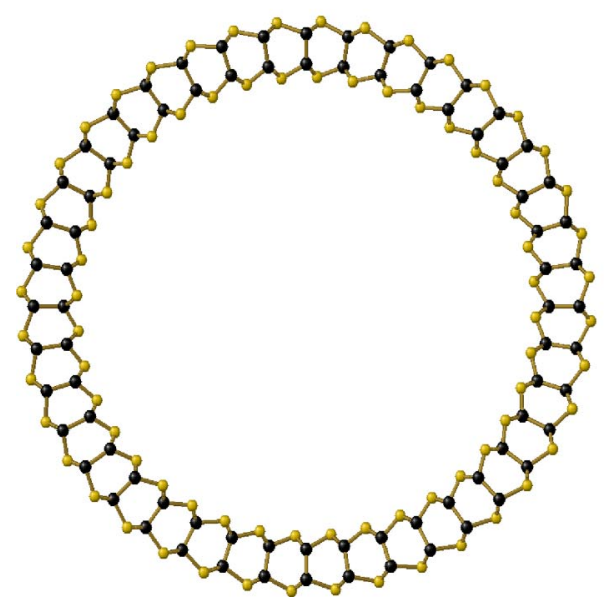

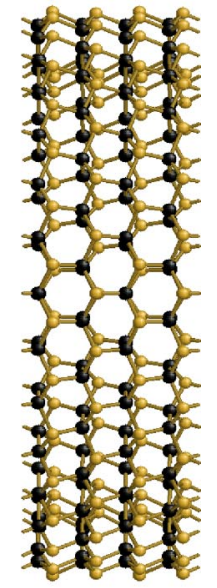

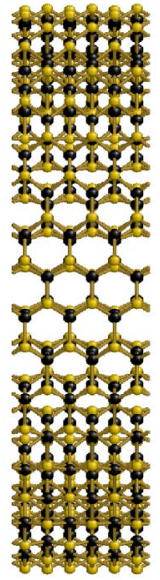

FIG. 2. Structure of $(30,0)$ and $(20,20)$ gallium sulfide $(\mathrm{GaS})$ nanotubes. On the left views down the axis of the nanotubes are shown; the right images show a side view in each case.

which initial-guess tubular structures have been fully relaxed with respect to atomic positions and tube cell length. Initial configurations of the nanotubes were constructed by mapping a 2D sheet of double-layered hexagonal $\mathrm{GaS}$ with above given bond lengths onto a cylinder. In labeling these tubes we adopt the convention of Ref. 21 . We have considered both armchair nanotubes $(n, n)$ with $n \in[18,30]$ and zigzag nanotubes $(n, 0)$ with $n \in[30,40]$. The lower bounds of the intervals are determined by the fact that we find stable tubular structures only for mean diameters larger than $36 \AA$. Smaller tubes became unstable due to strong distortions of $\mathrm{Ga}-\mathrm{S}$ bonds at the inner and outer surfaces of the tube. For each nanotube thus constructed, a set of structural relaxation calculations were performed, each one imposing a different axial strain on the tube, with the aim of finding the atomic configuration and lattice parameter of minimum energy. Structural relaxation was performed using the conjugate gradient technique ${ }^{22}$ in which all atoms were allowed to relax, and no constraints were imposed on the system. Figure 2 illustrates the minimum-energy structures found for gallium sulfide $(30,0)$ and $(20,20)$ nanotubes, respectively. The two structures shown are representative of those found for all the other tubes considered in this work, which differ from these only in mean diameter $\bar{D}$ and thus in the number of $\mathrm{Ga}_{3} \mathrm{~S}_{3}$ rings around the section of the tube. The main results of our simulations are summarized in Table I. The above results indicate the possibility of the existence of stable tubular gallium sulfide structures. This is strongly supported by the calculation of the strain energy $E_{S}$ of $\mathrm{GaS}$ nanotubes. The strain energy is the energy difference between a tube and the planar structure it is based on. It is the work that has to be performed to change the curvature radius $R$ from planar ( $R$ $=\infty$ ) to cylindrical (finite $R$ ) geometry. Plotting the strain energies as function of the mean diameter $\bar{D}$, shown in Fig. 3 , they all converge roughly as $\alpha \bar{D}^{-2}$ towards the value of the reference structure (infinite planar $\mathrm{GaS}$ sheet, $E_{\mathrm{S}}=0$ ) as the diameter $\bar{D}$ is increased, which can be understood easily from elasticity theory. ${ }^{23}$ For our calculations the $\alpha$ parameter has the value $1.413 \mathrm{eV} \mathrm{nm}^{2} /$ atom. The planar hexagonal

TABLE I. Selected properties of gallium sulfide nanotubes as obtained from our DFTB calculations.

\begin{tabular}{|c|c|c|c|c|c|c|c|c|c|}
\hline & & $D_{0}^{\min }(\AA)$ & $D_{0}^{\max }(\AA)$ & $\bar{D}_{0}(\AA)$ & $E_{S}(\mathrm{eV} /$ atom $)$ & $E_{\text {gap }}(\mathrm{eV})$ & $Y_{S}\left(\mathrm{GPa}^{*} \mathrm{~nm}\right)$ & $Y(\mathrm{GPa})$ & $\sigma$ \\
\hline \multirow[t]{6}{*}{$\mathrm{GaS}(n, 0)$} & $(30,0)$ & 32.14 & 41.04 & 36.59 & 0.1065 & 2.571 & 89.6 & 263.5 & 0.274 \\
\hline & $(32,0)$ & 34.39 & 43.32 & 38.85 & 0.0945 & 2.723 & 90.2 & 265.2 & 0.254 \\
\hline & $(34,0)$ & 36.48 & 45.63 & 41.15 & 0.0844 & 2.856 & 92.6 & 272.4 & 0.219 \\
\hline & $(36,0)$ & 38.98 & 47.94 & 43.46 & 0.0757 & 2.975 & 92.2 & 271.3 & 0.246 \\
\hline & $(38,0)$ & 41.25 & 50.22 & 45.73 & 0.0683 & 3.077 & 92.3 & 271.7 & 0.291 \\
\hline & $(40,0)$ & 43.58 & 52.56 & 48.07 & 0.0619 & 3.166 & 94.0 & 276.7 & 0.280 \\
\hline \multirow[t]{7}{*}{$\operatorname{GaS}(n, n)$} & $(18,18)$ & 33.23 & 42.15 & 37.69 & 0.0984 & 2.615 & 92.2 & 271.4 & 0.379 \\
\hline & $(20,20)$ & 37.18 & 46.12 & 41.65 & 0.0805 & 2.867 & 91.6 & 269.5 & 0.401 \\
\hline & $(22,22)$ & 41.16 & 50.13 & 45.64 & 0.0669 & 3.069 & 93.5 & 275.0 & 0.278 \\
\hline & $(24,24)$ & 45.18 & 54.15 & 49.66 & 0.0564 & 3.237 & 94.2 & 277.1 & 0.282 \\
\hline & $(26,26)$ & 49.18 & 58.17 & 53.67 & 0.0481 & 3.377 & 94.4 & 277.6 & 0.285 \\
\hline & $(28,28)$ & 53.18 & 62.19 & 57.69 & 0.0415 & 3.501 & 97.4 & 286.5 & 0.286 \\
\hline & $(30,30)$ & 57.25 & 66.27 & 61.76 & 0.0361 & 3.592 & 96.8 & 284.7 & 0.291 \\
\hline
\end{tabular}




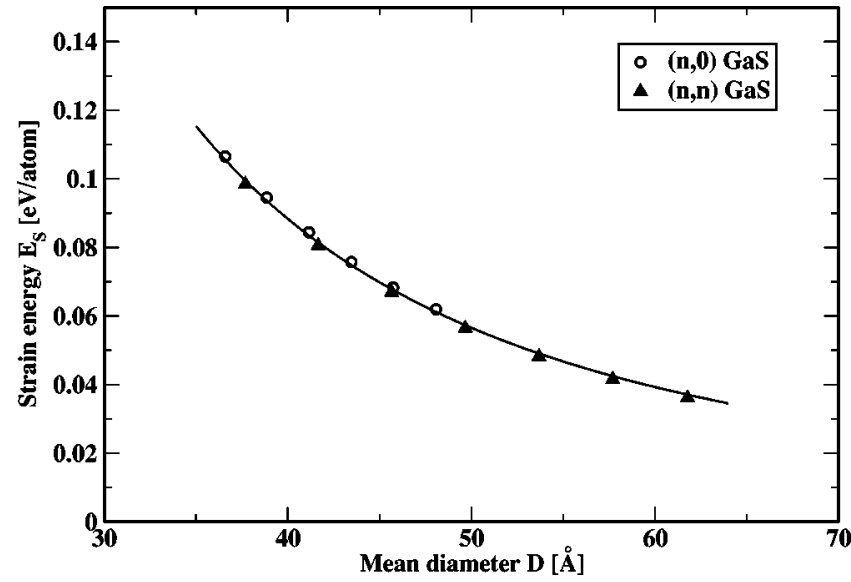

FIG. 3. Strain energies of $(n, n)$ and $(n, 0)$ gallium sulfide $(\mathrm{GaS})$ nanotubes as a function of the mean tube diameter. The straight line is the best $1 / D^{2}$ fit, see text.

$\mathrm{GaS}$ sheet as well as all the nanotubes considered here were found to be semiconducting with a direct band gap. In our calculations the band gap $E_{\mathrm{G}}$ in the hexagonal GaS sheet was found to be $4.74 \mathrm{eV}$. Figure 4 shows the gap size $E_{\mathrm{G}}$ of nanotubes as function of mean diameter $\bar{D}$. We find that the gap size grows chirality independent towards the value of flat $\mathrm{GaS}$ sheet as the tube diameter is increased in a nearly $E_{\mathrm{G}}$ $=4.74-\beta / \bar{D}$ law $(\beta=76.8 \mathrm{eV} \AA$ ). The discussed behavior is clearly in contrast to carbon nanotubes (NT's), where the band gap is strongly varying with diameter and chirality of the tubes, but similar to $\mathrm{MoS}_{2}$ NT's. The crystal structure of layered $\mathrm{GaS}$ is closely related to that of $\mathrm{MoS}_{2}$. For nanotubes of $\mathrm{MoS}_{2}$ we had predicted ${ }^{25}$ also a reduction of the gap size with decreasing diameter due to the effect of the curvature of the S-Mo-S triple layer. This effect has been recently confirmed experimentally. ${ }^{26}$ Such curvature effect can explain the size trend of the gap also in the double-layered GaS case.

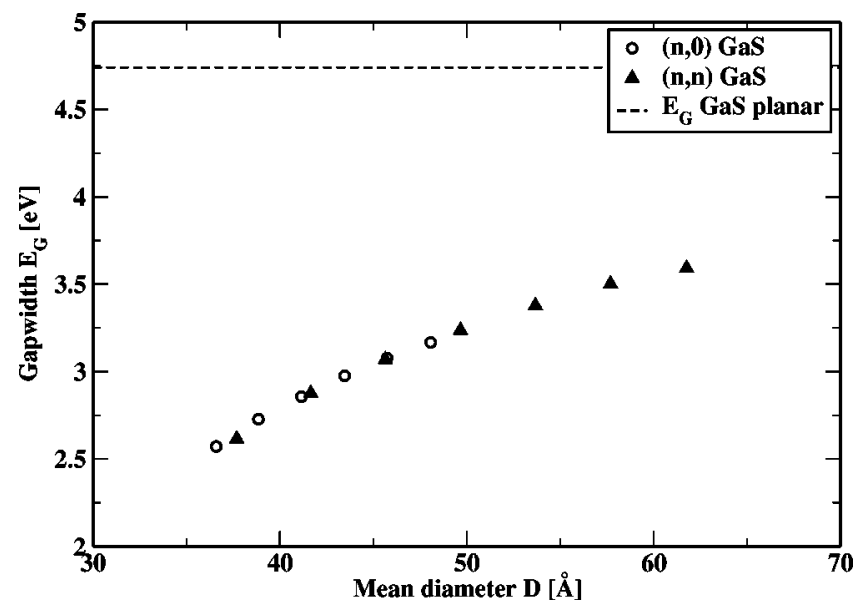

FIG. 4. Gap size vs mean diameter for proposed gallium sulfide (GaS) nanotubes. The optical gap of planar GaS structure is symbolized by a single line at $4.74 \mathrm{eV}$. Due to a limited basis set we overestimate the gap size somewhat. For comparison: The fundamental absorption edges in $\mathrm{GaS}$ give a gap size of $3.3 \mathrm{eV}$ for layered GaS (Ref. 24).

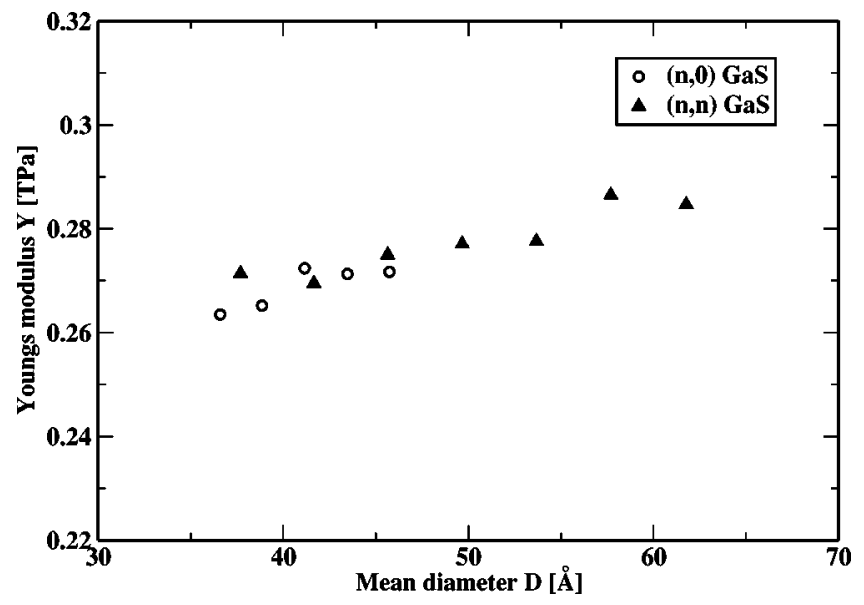

FIG. 5. The $Y$ modulus of $(n, n)$ and $(n, 0)$ gallium sulfide $(\mathrm{GaS})$ nanotubes as a function of the mean tube diameter.

Additionally, we studied the mechanical properties of the proposed gallium sulfide GaS nanotubes. We have calculated the Young's modulus $Y$ and the Poisson ratio $\sigma$ of these tubes, drawn in Figs. 5 and 6, respectively. Following the discussions of Hernandez, ${ }^{27}$ we define also in case of GaS tubes two elastic moduli

$$
Y=\left.\frac{1}{V_{0}}\left(\frac{\partial^{2} E}{\partial \varepsilon^{2}}\right)\right|_{\varepsilon=0}, \quad Y_{S}=\left.\frac{1}{S_{0}}\left(\frac{\partial^{2} E}{\partial \varepsilon^{2}}\right)\right|_{\varepsilon=0}
$$

by monitoring the changes in energy under applied axial stress $\left[\varepsilon=\left(L-L_{0}\right) / L_{0}\right]$. The first equation, the classical definition of Young's modulus containing the equilibrium volume $V_{0}$ of the tube, for a hollow cylinder with length $L_{0}$ and radius $R$ yields $V_{0}=2 \pi L_{0} R \delta R$, depending on an unknown thickness $\delta R$ of the tube. To compare mechanical strengths of various nanotubes one mostly uses $\delta R$ $=0.34 \mathrm{~nm}$, the interlayer spacing of graphite, as we do here. The second definition of $Y_{S}$ overcomes this problem by using only the equilibrium surface area $S_{0}$ of the tube. Both values are connected by $Y=Y_{S} / \delta R$, and are shown in Table

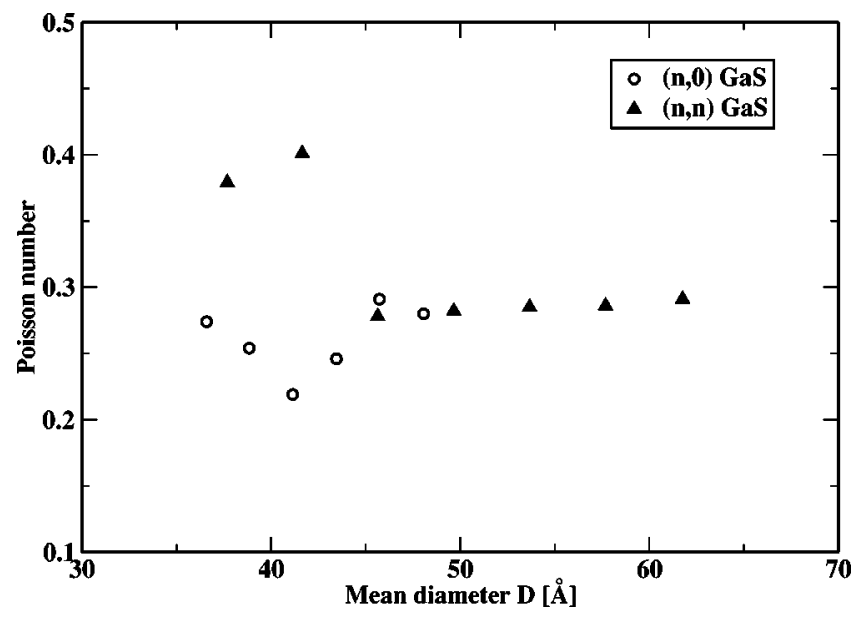

FIG. 6. The Poisson ratio $\sigma$ of $(n, n)$ and $(n, 0)$ gallium sulfide $(\mathrm{GaS})$ nanotubes as a function of the mean tube diameter. 
I. In comparison to Ref. 27 we clearly see that GaS-based nanotubes are less stiffer than other types of nanotubes hitherto considered, such as $\mathrm{C}, \mathrm{BN}, \mathrm{BC}_{3}, \mathrm{BC}_{2} \mathrm{~N}$ nanotubes, but that their Young's modulus is in the range that could have been expected from the mechanical properties of bulk GaS. For pure carbon single-walled nanotubes (SWNT's) we get indeed $1.20 \mathrm{TPa}$ for a $(20,0) \mathrm{SWNT}$ within the same DFTB method. The Poisson number $\sigma$ measures the relative change in diameter (mean diameter $\bar{D}$ ) under axial stress and is defined as

$$
\left(\bar{D}-\bar{D}_{0}\right) / \bar{D}_{0}=-\sigma \varepsilon,
$$

where $\bar{D}_{0}$ is the mean diameter at equilibrium. The Poisson ratios $\sigma$ of $\mathrm{GaS}$ nanotubes are within the same range of above-mentioned carbon based composite nanotubes, see
Table I and Ref. 27 again. As example we calculated the DFTB $\sigma$ values for $(20,0)$ and $(20,20)$ carbon SWNT's as 0.288 and 0.283 , respectively. As technical detail we mention that our $\sigma$ value is an average value over changes in mean diameter $\bar{D}$ under different small applied stresses.

Summarizing, our calculations show that stable GaS nanotubes are energetically favorized structures and therefore could exist. All gallium sulfide GaS nanotubes, proposed in this paper have a stable semiconducting gap, independent of their chirality and converges rapidly with increasing diameter to that of the 2D layer. Our findings are also supported by the observation of tubular structures in the catalyzed growth of InS. ${ }^{28}$

The authors thank R. Tenne for useful suggestions. The work was partially supported by the German-Israel foundation (GIF).
${ }^{1}$ S. Iijima, Nature (London) 354, 56 (1991).

${ }^{2}$ Carbon Nanotubes, edited by M. S. Dresselhaus, G. Dresselhaus, and $\mathrm{Ph}$. Avouris (Springer-Verlag, Berlin, 2001).

${ }^{3}$ E.T. Mickelson, C.B. Hoffmann, A.G. Rinzler, R.E. Smalley, R.H. Hauge, and J.L. Margrave, Chem. Phys. Lett. 296, 188 (1998).

${ }^{4}$ G. Seifert, Th. Köhler, and Th. Frauenheim, Appl. Phys. Lett. 77, 1313 (2000).

${ }^{5}$ B.W. Smith, M. Monthrioux, and D.E. Luzzi, Nature (London) 396, 323 (1998).

${ }^{6}$ R. Tenne, L. Margulis, M. Genut, and G. Hodes, Nature (London) 365, 444 (1992).

${ }^{7}$ G. Seifert, H. Terrones, M. Terrones, G. Jungnickel, and Th. Frauenheim, Phys. Rev. Lett. 85, 146 (2000).

${ }^{8}$ Y. Li, J. Wang, Z. Deng, Y. Wu, X. Sun, D. Yu, and P. Yang, J. Am. Chem. Soc. 123, 9904 (2001).

${ }^{9}$ W. Han, S. Fan, Q. Li, and Y. Hu, Science 277, 1287 (1997).

${ }^{10}$ M. Terrones, A.M. Benito, C. Mantega-Digo, W.K. Hsu, O.I. Osman, J.P. Hare, D.G. Reid, H. Terrones, A.K. Cheetham, K. Prassides, H.W. Kroto, and D.R.M. Walton, Chem. Phys. Lett. 257, 576 (1996).

${ }^{11}$ M. Nath and C.N.R. Rao, J. Am. Chem. Soc. 123, 4841 (2001).

${ }^{12}$ M.E. Spahr, P. Bitterli, R. Nesper, F. Krumeich, and H.U. Nissen, Angew. Chem., Int. Ed. 37, 1263 (1998).

${ }^{13}$ M. Cote, M.L. Cohen, and D.J. Chadi, Phys. Rev. B 58, R4277 (1998).

${ }^{14}$ A.N. MacInnes, M.B. Power, and A.R. Barron, Chem. Mater. 4, 11 (1992).

${ }^{15}$ A.N. MacInnes, M.B. Power, and A.R. Barron, Chem. Mater. 5, 1344 (1993).
${ }^{16}$ A.N. MacInnes, M.B. Power, A.R. Barron, P.P. Jenkins, and A.F. Hepp, Appl. Phys. Lett. 62, 711 (1993).

${ }^{17}$ Th. Frauenheim, G. Seifert, M. Elstner, Z. Hajnal, G. Jungnickel, S. Suhai, and R. Scholz, Phys. Status Solidi B 227, 41 (2000).

${ }^{18}$ D. Porezag, Th. Frauenheim, Th. Köhler, G. Seifert, and R. Kaschner, Phys. Rev. B 51, 12947 (1995).

${ }^{19}$ G. Seifert, Th. Köhler, H.M. Urbassek, E. Hernandez, and Th. Frauenheim, Phys. Rev. B 63, 193409 (2001).

${ }^{20}$ G. Seifert, Th. Köhler, and Th. Frauenheim, Appl. Phys. Lett. 77, 1313 (2000).

${ }^{21}$ M.S. Dresselhaus, G. Dresselhaus, and R. Saito, Phys. Rev. B 45, 6234 (1992).

${ }^{22}$ W. H. Press, S. A. Teukolsky, W. T. Vetterling, and B. P. Flannery, Numerical Recipes in Fortran, 2nd ed. (Cambridge University Press, Cambridge, 1992).

${ }^{23}$ G.G. Tibbets, J. Cryst. Growth 66, 632 (1983).

${ }^{24}$ J.L. Brebner and G. Fischer, Can. J. Phys. 41, 561 (1963).

${ }^{25}$ G. Seifert, in Electronic Properties of Novel Material-Molecular Nanostructures, edited by Hans Kuzmany, Jörg Fink, Michael Mehring, and Siegmar Roth, AIP Conf. Proc. No. 544 (AIP, Melville, NY, 2000), p. 415.

${ }^{26}$ L. Scheffer, R. Rosentzweig, A. Margolin, R. Popovitz-Biro, G. Seifert, S.R. Cohen, and R. Tenne, Phys. Chem. Chem. Phys. 4, 2095 (2002).

${ }^{27}$ E. Hernandez, C. Goze, P. Bernier, and A. Rubio, Appl. Phys. A: Mater. Sci. Process. A68, 287 (1999).

${ }^{28}$ J.A. Hollingsworth, D.M. Poojary, A. Clearfield, and W.E. Buhro, J. Am. Chem. Soc. 122, 3562 (2000). 